\title{
Eine Frage des Standpunktes
}

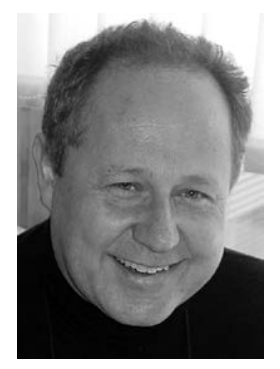

Manfred Wildner
Korrespondenzadresse

Prof. Dr. med. Manfred Wildner

Bayerisches Landesamt für Gesundheit und

Lebensmittelsicherheit

Veterinärstraße 2

85764 Oberschleißheim

Manfred.Wildner@Igl.bayern.de

\section{Bibliografie}

DOI https://doi.org/10.1055/a-0924-9655

Gesundheitswesen 2019; 81: 463-464

(c) Georg Thieme Verlag KG Stuttgart · New York

ISSN 0941-3790
„Gib mir einen Punkt, wo ich hintreten kann und ich bewege die Erde" - so lautet ein dem griechischen Mathematiker, Physiker und Ingenieur Archimedes im Zusammenhang mit dem Hebelgesetz zugeschriebener Satz [1]. Als ,archimedischen Punkt“ bezeichnen wir seither auch viel allgemeiner einen festen Punkt, wie z. B. eine offensichtliche, unbezweifelbare Wahrheit in einer Argumentation. Auch wenn wir im Gesundheitswesen nicht unbedingt die Welt aus den Angeln heben wollen, so ist doch eine gute Ortskenntnis, im Sinne eines guten Verständnisses der Gegebenheiten, Zuständigkeiten und Regularien, ebenfalls von großer Bedeutung.

Damit wird der Standpunkt auch zur (gesundheits)politischen Frage. Eine Definition von Politik als „die Regelung der Angelegenheiten eines Gemeinwesens durch verbindliche Entscheidungen “ [2] ist dabei nur eine erste Annäherung an den schillernden, facettenreichen Begriff „Politik“ und bedarf weiterer Differenzierungen. In den angloamerikanischen Politikwissenschaften wird bereits sprachlich eine feinere Unterteilung des Politikbegriffs vorgenommen: währende policy die inhaltlichen und strategischen politischen Ausrichtungen meint, bezieht sich die Bezeichnung politics auf - teilweise auch taktisch motivierte - politische Aushandlungsprozesse. Polity hingegen bezieht sich auf strukturelle und institutionelle Aspekte wie z. B. die gesetzlich vorgegebenen Organe und Verfahrenswege, innerhalb derer politische Inhalte ver- und ausgehandelt werden.

Im Gesundheitswesen sind solche gesetzlich vorgegebenen Verortungen von Entscheidungs- und Aushandlungsprozessen allgegenwärtig und von größter Bedeutung, das Wissen um die „polity“- Aspekte damit unverzichtbar. Diese politischen Verortungen gründen sich zum einen in der föderalen Ordnung der Bundesrepublik als demokratischem und sozialem Bundesstaat (Art. 20 Grundgesetz). Dabei finden sich auf der Bundesebene die Zuständigkeiten für die verschiedenen Sozialgesetzbücher und die damit auch geschaffenen Körperschaften und Institutionen: die gesetzliche Kranken- und Pflegeversicherung, die Unfallversicherung, die Rentenversicherung. Hinzu kommen direkte staatliche Verwal- tungsorgane wie die obersten (Ministerien) und oberen Bundesbehörden, z. B. Robert Koch-Institut (RKI), Bundesinstitut für Arzneimittel und Medizinprodukte (BfArM), Bundeszentrale für gesundheitliche Aufklärung (BZgA), aber auch weitere Institutionen mittelbarer staatlicher Verwaltung wie der Gemeinsame Bundesausschuss (G-BA), das Institut für Qualität und Wirtschaftlichkeit im Gesundheitswesen (IQWiG), das Institut für Qualitätssicherung und Transparenz im Gesundheitswesen (IQTiG) u.v.a.m. Auf Länderebene betreffen die gegebenen Zuständigkeiten beispielsweise Forschung und Lehre, die Organisation des Öffentlichen Gesundheitsdienstes, Krankenhausplanungen und sektorenübergreifende Versorgungsfragen (Landesgremium nach §90a SGB V). Am bürgernächsten ist letztlich die kommunale Ebene mit Ihren Aufgaben der Daseinsfürsorge, den von den Bezirken getragenen Kliniken, den regionalen Gremien wie bspw. den psychosozialen Arbeitsgemeinschaften und den diversen Gesundheitskonferenzen [3].

Diese Verortungen spiegeln sich, teilweise in Folge der föderalen Ordnung, auch in den institutionellen Interessen und Leitideen. Beispiele dafür sind die nicht selten divergierenden Standpunkte von Leistungserbringern in der stationären und ambulanten Versorgung und den Financiers dieser Leistungen, verkörpert z. B. durch die gesetzlichen und privaten Krankenversicherungen, welche sich wiederum von den Präferenzen auf Seiten der Versicherten bzw. Patienten unterscheiden können. Zusätzlich lassen sich auch innerhalb solcher Akteursgruppen weitere Standpunkte differenzieren: bei den Leistungserbringern die Interessen der unterschiedlichen Facharztgruppen, z. B. bei den Verhandlungen der Zuordnung von Punktwerten im ambulanten Bereich oder von Räumlichkeiten und Ressourcen im stationären Bereich, bei den Financiers der politisch beförderte Wettbewerb zwischen den Kassen und nicht zuletzt finden sich auch unterschiedliche Interessen und Präferenzen bei den Versicherten bzw. Patientengruppen selbst, auch abhängig vom jeweils eigenen Gesundheitszustand oder der jeweils gegebenen finanziellen Leistungsfähigkeit. 
Und während bspw. wissenschaftlich oder unternehmerisch geprägte Akteure auf Innovation, Erneuerung und Änderung im Sinne einer erhofften Verbesserung drängen und dafür auch bereit sind, Risiken einzugehen, sind Entscheidungsträger in Politik und Körperschaften öffentlichen Rechts oft konservativ und risikoavers: das Streben nach Erfolg ist eben doch etwas anderes als das Vermeiden von Misserfolg. Gleichzeitig kann, themenabhängig, auch das Gegenteil der Fall sein: z.B. das langjährige politische, in weiten Bereichen erfolglose, Ringen um Fortschritte in der Digitalisierung und damit auch um mehr Effizienz und Transparenz im deutschen Gesundheitswesen ist ein Geschehen, dass mancherorts gar nicht verstanden würde. Ein geografisch zu verortendes Gegenbeispiel ist bspw. Estland mit seiner fortgeschrittenen digitalen Infrastruktur [4].

Deutlich wird so die erhebliche Komplexität und teilweise Vielfachsteuerung im „System Gesundheitswesen“ [5] mit einer Beeinflussung der vielfältigen Standpunkte durch Regionalität, Professionalität, institutionelle und persönliche Interessen. So wundert es auch nicht, dass der Erfolg bzw. Misserfolg von Maßnahmen in hohem Maße eben nicht nur von der Maßnahme selbst, sondern auch von den verschiedenen Kontexten der (versuchten) Umsetzung mit beeinflusst wird. Diese Kontextsensitivität trägt noch einmal erheblich zur Komplexität im Gesundheitswesen bei. „Gib mir einen festen Punkt..." - dieser Seufzer wird in diesem politischen und argumentativen Ringen wohl des Öfteren im Stillen formuliert worden sein. Die Auflösung wiederstreitender Interessen benötigt daher dezidiert auch strukturelle Unterstützungen im polity-Sinn: Institutionen und institutionalisierte Vermittlungsformen, welche mit legitimiertem Mandat schlichtend und gestaltgebend und unter Wahrung der Verhältnismäßigkeit unterstützen und ermöglichen. Dies kann als eine flankierende Handlungsoption auch ein beständiges Aussieben von gesundheitspolitischen und pseudowissenschaftlichen „Fake News“ beinhalten sowie als eine weitere beispielhafte Handlungsoption eine nachhaltige Anwaltschaft mit langfristiger Perspektive bezüglich verschiedener Verteilungsaspekte.

Diese Sensibilität für regionale und institutionelle Verortungen und Standpunkte spiegeln und leben auch die Beiträge dieser Ausgabe: Zum Nationalen Aktionsplan Gesundheitskompetenz und seiner Notwendigkeit, seinen Zielen und Inhalten, zur Inanspruchnahme der ambulanten Notfallversorgung in Westfalen-Lippe mithilfe einer medizingeografischen Studie, zur Bereitschaft zur Teilnahme an der ambulanten spezialfachärztlichen Versorgung (ASV), zur ambulanten Notfallversorgung von Pflegeheimbewohner aus pflegerischer Sicht, ebenso der Beitrag zu Family Health Teams in Ontario, die Vorstellung eines kanadischen Primärversorgungsmodells mit Anregungen auch für Deutschland, die Befragung der Bevölkerung zur hausärztlichen Versorgungssituation und zur Einführung neuer Versorgungsformen in schwer zu versorgenden Regionen, die systematische Übersicht zur Epidemiologie des Dekubitus in Deutschland sowie die Ergebnisse einer Befragung von Hausarztpatienten zu Patientenverfügungen in Hausarztpraxen.
Der praktische Nutzen solcher Überlegungen zu Orten, Verortungen und damit ein Stück weit vorgegebenen Standpunkten? Die Frage nach dem „archimedischen Punkt“, einem festen und sicheren Boden als Grundlage eines Standpunktes ist nur ein Aspekt. Häufig bestimmt auch unsere institutionelle Verortung unsere Sichtweise: wo wir sitzen, d. h. wo unser Schreibtisch steht, bestimmt oft auch, wo wir argumentativ stehen. Die unterschiedlichen Standpunkte zu kennen und zu erkennen, ist eine Notwendigkeit, die keinem und keiner im Gesundheitswesen Tätigen erspart bleibt und gleichsam die Voraussetzung dafür ist, wirksam den einen oder anderen Hebel anzusetzen. Dieses Erkennen sollte dabei auch eine Reflexion der eigenen impliziten und expliziten Standpunkte beinhalten: Auch unser Schreibtisch hat seinen Ort. Institutionelle Interessen sind legitim, allerdings nicht absolut zu setzen und so hat der Hebel, den wir ansetzen wollen oder sollen, nicht nur den technischen Aspekt der Wirksamkeit, sondern in seiner Hebelrichtung und Motivation auch eine moralische Dimension. Um etwas, im Sinne „guter Stewardship“ [6], auch in die „richtige“ Richtung bewegen zu können, bedarf es neben den Institutionen und Verfahren, gerade im Gesundheitswesen, als archimedischem Punkt v. a. auch tragfähiger, transparenter und akzeptierter gemeinsamer Stand- und Eckpunkte. Standpunkte, welche sich mit dem Anspruch von Humanität, neben Qualität und Wirtschaftlichkeit ( $\$ 70$ SGB V), vor allem auch in den gesellschaftlich verbindenden Werten gründen, welche menschliche Gemeinschaft erst ermöglichen. Soziale Verantwortung, Mitgefühl und Reziprozität als Grundlagen von Fairness und Würde sollten im Gesundheitswesen als quasi-archimedische Standpunkte keine Frage des Standpunktes sein [7-9].

\section{Literatur}

[1] Pappos von Alexandrien. Synagoge (Sammlung), Buch VIII;

[2] Fuchs D, Roller E, Politik. InFuchs D, Roller E., (Hrsg.). Lexikon Politik. Hundert Grundbegriffe; Reclam, Stuttgart: 2009: S 205-209

[3] Hollederer A. Gesundheitskonferenzen in Deutschland: ein Überblick. Gesundheitswesen 2015; 77: 161-167

[4] Bertelsmann-Stiftung. Estland: Spitzenreiter für Digital Health URL https://www.bertelsmann-stiftung.de/de/unsere-projekte/der-digitale-patient/projektthemen/smarthealthsystems/estland/, download 04.06.2019

[5] Alber ]. Das Gesundheitswesen der Bundesrepublik Deutschland. Entwicklung, Struktur und Funktionsweise. Frankfurt am Main: Campus Verlag 1992; 14

[6] Saltman RB, Ferroussier-Davis O. The concept of stewardship in health policy. Bulletin World Health Org 2000; 78: 732-739

[7] Rawls ]. Eine Theorie der Gerechtigkeit. Suhrkamp, Frankfurt am Main 1979, (englisch: A Theory of Justice. Cambridge, MA: Harvard University Press, 1971/1999;

[8] Bieri P. Eine Art zu leben. Frankfurt a. M.: Fischer Verlag 2015;

[9] De Waal F. Moral behaviour in animals. URL https://www.ted.com/ talks/frans_de_waal_do_animals_have_morals/transcript\#t-2420 download 04.06.2019 